\title{
Direct-acting antiviral agents in the therapy of hepatitis $C$ virus-related mixed cryoglobulinaemia: a single-centre experience
}

\author{
Gianfranco Lauletta*, Sabino Russi, Fabio Pavone, Angelo Vacca and Franco Dammacco
}

\begin{abstract}
Background: The efficacy and safety of direct-acting antiviral agents (DAAs) were evaluated in a cohort of prospectively enrolled patients with hepatitis C virus (HCV)-related mixed cryoglobulinaemia (MC), an immune complex-mediated vasculitis of small and medium vessels in which the pathogenetic role of HCV has been clearly established.

Methods: Twenty-two patients received DAAs. Clinical and laboratory features were recorded at baseline, every 4 weeks until the end of treatment (EoT), and 12 weeks afterwards. Primary efficacy endpoints were (a) sustained virological response 12 weeks after therapy completion (SVR12), (b) regression of symptomatology (clinical response) and (c) cryoglobulin disappearance or cryocrit reduction $\geq 50 \%$ (immunological response). Complete response (CR) was defined as the occurrence of all three primary endpoints; partial response (PR) was defined as the occurrence of SVR12, with or without either immunological or clinical response; and no response was defined as missing the achievement of all three endpoints.

Results: All patients reached SVR12. Compared with basal values, mean cryocrit values were significantly decreased at EoT and SVR12. A significant reduction of alanine transaminase and a parallel increase of complement component C4 levels were also detected. Rheumatoid factor activity was significantly reduced at EoT but not at SVR12. At SVR12, a CR was established in 14 patients (63.7\%) and a PR in 8 patients (36.3\%). In one patient with small lymphocytic lymphoma, the tumour progressed despite viral clearance. Mild adverse events were recorded in nine patients (40.9\%).

Conclusions: The response rates induced by the use of DAAs in patients with MC were remarkably higher than those previously achieved with pegylated interferon-a/ribavirin, with or without rituximab. A much longer follow-up is desirable to achieve useful information in terms of persistent viral clearance and clinical response.
\end{abstract}

Keywords: Cryoglobulinaemia, Direct-acting antiviral agents, Hepatitis C virus, Rheumatoid factor

\section{Background}

Hepatitis $\mathrm{C}$ virus (HCV) is a major cause of chronic liver disease that can potentially progress to cirrhosis and hepatocellular carcinoma (HCC) [1-3]. It has been estimated that about 130 million to 150 million people worldwide have $\mathrm{HCV}$ infection and that HCV causes about 500,000 deaths each year [4]. In addition to liver damage, HCV infection is associated with several extrahepatic disorders [5]; among them, the striking association between $\mathrm{HCV}$

\footnotetext{
* Correspondence: gianfranco.lauletta@uniba.it

Department of Biomedical Sciences and Human Oncology, University of Bari "Aldo Moro", Piazza G. Cesare, 11-70124, Bari, Italy
}

infection and mixed cryoglobulinaemia $(\mathrm{MC})$, an immune complex-mediated vasculitis of small and medium vessels, has been clearly established [6,7]. On the basis of B-cell clonal expansion that can be detected in the majority of patients, $\mathrm{MC}$ can also be considered a low-grade, indolent lymphoproliferative disorder [8].

Clinically, MC is characterised by the classic Meltzer's triad of symptoms (purpura, weakness and arthralgia). The clinical spectrum of MC is quite variable, however, ranging from skin lesions, including recurrent purpuric eruptions, leg dyschromatosis and torpid ulcers, to peripheral neuropathy and renal damage. In a minority of 
patients, multi-organ involvement may result in lifethreatening conditions $[9,10]$. In our experience, compared with patients with chronic HCV infection without cryoglobulinaemia, HCV-positive patients with MC exhibit a lower rate of liver fibrosis and a lower cumulative probability of developing cirrhosis and $\mathrm{HCC}$, but a more frequent occurrence of renal failure, neurologic impairment and B-cell non-Hodgkin lymphoma (B-NHL). Even so, the 15-year survival rates in these groups have been found to be roughly similar [11].

The therapeutic approach to MC has conventionally been based on the use of steroids and immunosuppressive drugs. Interferon (IFN)-based treatment was introduced in 1987, before the identification of HCV as the major etiologic factor [12, 13]. Subsequently, the antiviral combination of pegylated interferon- $\alpha$ plus ribavirin (pIFN- $\alpha+\mathrm{RBV}$ ) was employed to obtain a sustained virological response (SVR), and for many years, this combination has been considered the standard of care for the treatment of chronic $\mathrm{HCV}$ infection, capable of inducing SVR and clinical remission of vasculitis in about $78 \%$ of patients with MC [14, 15]. A multicentre, open-label study showed viral response rates ranging from $36 \%$ to $64 \%$ according to viral genotype and a clinical response in $88 \%$ of the patients [16]. The association of rituximab (RTX), an anti-CD20 monoclonal antibody aimed at inducing an effective B-cell depletion, resulted in further benefits, both in patients refractory to antiviral therapy $[17,18]$ and in those previously untreated [19]. In our experience, the addition of RTX to the PIFN- $\alpha+$ RBV combination has resulted in a $33 \%$ complete response (CR) (i.e., virological, immunological and molecular response) [19].

However, the efficacy of therapy in terms of viral clearance has remained unsatisfactory and is burdened with remarkable side effects. The use of first-generation protease inhibitors, such as boceprevir or telaprevir, associated with pIFN- $\alpha+\mathrm{RBV}$ in a triple-combination therapeutic regimen resulted in significantly higher response rates in patients with chronic $\mathrm{HCV}$ infection without and with MC. Serious adverse events were recorded in almost 50\% of patients, however [20].

The introduction of all-oral, direct-acting antiviral agents (DAAs) has dramatically changed the treatment of chronic $\mathrm{HCV}$ and consequently the prognosis of these patients. According to the viral molecular target, DAAs include inhibitors of non-structural proteins NS3/NS4 (simeprevir, paritaprevir and grazoprevir), NS5A (ledipasvir [LDV], daclatasvir [DCV], ombitasvir, elbasvir and velpatasvir) and NS5B (sofosbuvir [SOF] and dasabuvir) that are already available in Italy, except elbasvir, grazoprevir and velpatasvir, for which approval is pending. These drugs have been shown to be capable of eradicating the infection in over $90 \%$ of patients with chronic HCV, with limited variations related to viral genotype [21]. An additional advantage of DAAs is the possibility of avoiding IFN-related side effects. However, owing to the high costs of these drugs, it has been necessary to fix priority criteria among patients to be treated. Both the American and the European associations for the study of liver diseases, as well as the Italian Medicines Agency (AIFA), have established that extrahepatic manifestations of $\mathrm{HCV}$ infection, such as cryoglobulinaemic vasculitis and lymphoproliferative disorders, should be recognised as priority indications for treatment with DAAs [22, 23].

So far, only a few papers have been published on the efficacy and safety of the use of DAAs in patients with MC. Saadoun et al. [24] reported a 74\% rate of sustained virological response 12 weeks after therapy completion (SVR12) and an 87.5\% clinical response rate in 24 patients with $\mathrm{HCV}$-related MC treated with an SOF plus RBV combination. In another study, carried out with 12 patients with MC (7 of whom had renal involvement) treated with SOF-based regimens, the SVR12 rate was $83 \%$ and was associated with improvement in serum creatinine and reduction in proteinuria in patients with glomerulonephritis. In addition, an overall reduction or disappearance of cryoglobulins was recorded in $89 \%$ of the patients [25]. Mention should also be made of a prospective double-centre (Florence/Rome) study of a cohort of 44 consecutive patients with $\mathrm{HCV}$-associated MC, 2 of whom had evolved into an indolent NHL with monoclonal B-cell lymphocytosis. Following guidelinetailored therapy with DAAs, an SVR was demonstrated 12 and 24 weeks post-treatment in parallel with clinical response in all patients. A striking reduction in the mean cryocrit value was revealed at SVR12, and an even greater reduction was observed for sustained virological response 24 weeks after therapy completion (SVR24). A partial vasculitis response and a roughly $50 \%$ reduction of cryocrit were detected in the two patients with lymphoma. Adverse events were rather frequent but usually mild [26]. More recently, Bonacci et al. [27] reported a large prospective study of 64 cryoglobulinaemic patients with $\mathrm{HCV}$ infection, divided into those with symptomatic (55\% of cases) cryoglobulinaemia and those with asymptomatic cryoglobulinaemia (45\% of cases). Sixteen percent of the patients received an IFN-based DAA combination, whereas IFN-free regimens were administered to the remaining patients. Overall, 94\% of patients showed SVR12, and $71 \%$ achieved a clinical CR. In 10 of 13 patients, concomitant immunosuppressive therapy was reduced or withdrawn. Forty-eight percent of patients achieved an immunological response, defined as disappearance of circulating cryoglobulins and normalisation of complement and/or rheumatoid factor (RF). However, despite SVR12 in $52 \%$ of patients, cryoglobulins or complement 
consumption or RF activity persisted, a low baseline cryocrit level being the only factor associated with an immunological CR.

We report the results of our single-centre, prospective study of a cohort of patients with $\mathrm{MC}$ whose main features can be summarised as follows: (a) unresponsiveness to or relapse after the previous standard-of-care treatment consisting of pIFN- $\alpha+$ RBV combination, or previously untreated patients; (b) the assignment to receive variable combinations of DAAs according to the guidelines of the AIFA eligibility criteria; and (c) a post-treatment evaluation at weeks 12 and 24, with a prolonged follow-up until 12 months in some cases.

\section{Methods}

\section{Patients}

Twenty-two consecutive $\mathrm{HCV}$-positive patients with MC were enrolled in this study, with the aim being to assess both the efficacy and the safety of all-oral, IFN-free DAAs. Thirteen patients were non-responsive to or relapsed after previous pIFN- $\alpha+$ RBV combination therapy, whereas the remaining nine patients were treatmentnaïve. Written informed consent was obtained from all patients. Because treatments were administered on-label, no approval by the ethics committee was required, and the study was conducted according to the principles of the Declaration of Helsinki.

\section{Clinical and virological evaluation}

All patients were thoroughly examined according to validated criteria reported elsewhere [28]. Clinical features included the presence of purpura, fatigue and arthralgia, associated or not with skin ulcers. Renal damage and/or peripheral nervous system involvement were also looked for. Circulating cryoglobulins were detected as previously described [29-31] and immunochemically characterised by immunofixation, and their amounts were quantified as cryocrit (\%). Serum HCV RNA levels were assessed using the AmpliTaq real-time polymerase chain reaction system with a detection threshold of $15 \mathrm{IU} / \mathrm{ml}$ (Roche Molecular Systems Inc., Pleasanton, CA, USA), whereas HCV genotyping was performed using the VERSANT HCV genotype 2.0 assay (LiPA) (Siemens Healthcare Molecular Diagnostics, Berkeley, CA, USA).

\section{Treatment schedules}

Patients admitted to treatment had previously been examined by transient elastography (FibroScan; Echosens, Paris, France) for the assessment of liver fibrosis grade. Antiviral therapy was prescribed according to the AIFA eligibility criteria, HCV genotype, grade of liver fibrosis and prior treatment experience. For patients with RBV treatment, dose reduction was considered in accordance with technical schedule. The following regimens were employed: (a) daily SOF (400 mg) plus RBV (1000 to $1200 \mathrm{mg}$, depending on whether the patient's body weight was $\leq 75$ or $>75 \mathrm{~kg}$ ); (b) ombitasvir/paritaprevir/ritonavir (12.5 mg/75 mg/50 mg) plus dasabuvir (250 mg) (3D combo) twice daily, with or without weight-based RBV; (c) SOF/LDV (400 mg/90 mg) once daily; and (d) SOF (400 mg) plus DCV (60 mg) (SOF/DCV) once daily.

\section{Biochemical evaluation}

In addition to clinical examination, baseline evaluation included aspartate transaminase/alanine transaminase (ALT) values, haemoglobin level, platelet count, RF activity, serum levels of $\mathrm{C} 3$ and $\mathrm{C} 4$ complement component fractions, and serum protein electrophoresis. The same parameters were evaluated every 4 weeks during treatment and 12 weeks after therapy completion to establish whether SVR12 had been achieved.

\section{Endpoints}

Primary efficacy endpoints were (a) SVR12 (virological response), (b) regression of clinical manifestations of vasculitis (clinical response) and (c) cryocrit reduction by at least $50 \%$ or disappearance of cryoglobulins (immunological response). CR was defined as the occurrence of all primary endpoints, partial response (PR) as the occurrence of SVR12 with either immunological or clinical response, and no response (NR) as the lack of all criteria.

\section{Statistical analyses}

To assess the statistical power and significance of $C R$ proportion $\left(\pi_{1}\right)$, criterion power analysis was performed using G*Power 3.1 (Heinrich Heine University, Düsseldorf, Germany) with a one-tailed binomial test (one-sample case), assuming as the constant proportion the frequency of CR to pIFN- $\alpha+$ RBV therapy in patients with cryoglobulinaemia $\left(\pi_{0}=0.33\right)$ [19]. Sensibility (statistical power) was fixed at 0.80 . This was a one-tailed test because we assumed a greater antiviral efficacy of DAAs as compared with pIFN- $\alpha+\mathrm{RBV}$ therapy. To evaluate the frequencies and differences of virological and immunological responses, as well as $\mathrm{C} 4$ complement fraction, $\mathrm{RF}$ and ALT level normalisation, continuous variables were categorised as dichotomous variables and statistically analysed by cross-tabs and $\chi^{2}$ test.

Continuous variables were first subjected to descriptive statistical analyses to verify the normality of data (Kolmogorov-Smirnov test). The statistical significance of variable changes across the study was assessed by a general linear model (GLM) repeated measures analysis. Three factors (baseline, end of treatment [EoT] and SVR12 time points) and four variables (cryocrit, RF, C4 complement fraction and ALT levels) were taken into consideration. HCV RNA serum levels were excluded from the GLM because the test results for $\mathrm{HCV}$ were 
negative in all patients after treatment. The Huynh-Feldt test and group comparisons by simple contrasts were used for univariate repeated measures analysis of variance to evaluate EoT and SVR12 time point variations against the baseline. Two-tailed $\alpha$ probability ( $p$ value), estimation of the effect size in the sample $\left(\eta^{2}\right)$, and statistical power were reported, with $p<0.05$ being considered significant. Statistical analyses were performed by using IBM SPSS Statistics version 20.0 software (IBM, Armonk, NY, USA).

\section{Results}

Fourteen patients were treated with the SOF/RBV regimen for 12-24 weeks according to viral genotype and fibrosis stage, and seven patients with genotype 1 infection were treated with such a regimen before the availability of more effective combinations that all guidelines considered as optimal. Three patients received a $3 \mathrm{D}$ combo regimen, four with SOF/LDV and one SOF/DCV combination. Thirteen of twenty-two patients had experienced treatment failure in previous IFN-based therapeutic regimens. No statistically significant relationship was found between previous treatment failure and response to DAAs.

Table 1 summarises the main clinical, biochemical and virological characteristics of the patients enrolled. The subjects' mean age was 66.9 years, ranging from 46 to 84 , and the male/female ratio was $8 / 14$. All patients were viraemic with HCV RNA levels ranging from 2.1 to 7.7 logarithmic IU $/ \mathrm{ml}$. The mean cryocrit value was $1.8 \%$, ranging from $0.5 \%$ to $4 \%$. ALT levels ranged from 13 to $668 \mathrm{IU} / \mathrm{L}$ (mean \pm SD $104.8 \pm 144.7$ ), and C4 complement fraction ranged from 0 to $24 \mathrm{mg} / \mathrm{dl}$ (mean \pm SD 9.6 \pm 7.3 ). $\mathrm{RF}$ activity ranged from 0 to $530 \mathrm{IU} / \mathrm{ml}$ (mean $\pm \mathrm{SD}$ $69.3 \pm 119.1$ ). Cryoglobulins were immunochemically characterised as type $2 \mathrm{MC}$ (monoclonal immunoglobulin $\mathrm{M}$ [IgM]/polyclonal IgG) in 21 patients and as type $3 \mathrm{MC}$ (polyclonal IgM/polyclonal IgG) in the remaining 1 patient.

Meltzer's triad of symptoms (purpura, arthralgia and weakness) was consistently present in all patients. Cryoglobulinaemic glomerulonephritis was recorded in four patients (18.2\%) and severe peripheral neuropathy in two patients $(9.1 \%)$. Autoimmune haemolytic anaemia and idiopathic thrombocytopenic purpura were diagnosed in two patients each. In addition, four patients (18.2\%) showed a monoclonal IgG component (two with $\mathrm{k}$ light chains and two with $\lambda$ light chains) on serum protein electrophoresis, and three patients received a diagnosis of NHL, one with stage 4 small lymphocytic lymphoma (SLL) who received SOF/RBV therapy for 24 weeks followed by four RTX infusions at 1-week intervals, one with marginal zone B-NHL that extended to ocular adnexa previously treated with pIFN- $\alpha+\mathrm{RBV}+\mathrm{RTX}$, and one with diffuse centrocytic B-NHL who had received
Table 1 Baseline clinical, virologic and laboratory parameters of 22 patients with chronic hepatitis $C$ virus infection with mixed cryoglobulinaemia

\begin{tabular}{|c|c|}
\hline Parameters & Data \\
\hline Age, years, mean \pm SD (range) & $66.9 \pm 11.2(46-84)$ \\
\hline Female sex, $n(\%)$ & $14(63.6)$ \\
\hline Serum HCV RNA, logarithmic IU/ml, mean \pm SD & $6.02 \pm 1.2(2.1-7.7)$ \\
\hline \multicolumn{2}{|l|}{ HCV genotype, $n(\%)$} \\
\hline GT1 & $14(63.6)$ \\
\hline GT2 & $7(31.8)$ \\
\hline GT3 & $1(4.6)$ \\
\hline Cryocrit, \%, mean \pm SD (range) & $1.8 \pm 1.3(0.5-4)$ \\
\hline \multicolumn{2}{|l|}{ Cryoglobulin type, $n(\%)$} \\
\hline$\|$ & $21(95.4)$ \\
\hline III & $1(4.6)$ \\
\hline C3, mg/dl (n.v. 90-180) (mean \pm SD) & $93.4 \pm 15.3(51-118)$ \\
\hline C4, mg/dl (n.v. 10-40) (mean \pm SD) & $9.6 \pm 7.3(0-24)$ \\
\hline RF, IU/ml (n.v. 10-15) (mean \pm SD) & $69.3 \pm 119.1(0-530)$ \\
\hline \multicolumn{2}{|l|}{ Clinical features, $n(\%)$} \\
\hline Meltzer's triad & $22(100)$ \\
\hline Glomerulonephritis & $4(18.1)$ \\
\hline Peripheral neuropathy & $2(9.1)$ \\
\hline \multicolumn{2}{|l|}{ Liver involvement } \\
\hline ALT, IU/L (n.v. 12-78) (mean \pm SD) & $104.8 \pm 144.7(13-668)$ \\
\hline Cirrhosis, $n(\%)$ & $12(54.5)$ \\
\hline \multicolumn{2}{|l|}{ HCV-related tumours, $n(\%)$} \\
\hline Hepatocellular carcinoma & $2(10)$ \\
\hline Non-Hodgkin lymphoma & $1(5)$ \\
\hline Small lymphocytic lymphoma & $1(5)$ \\
\hline
\end{tabular}

Abbreviations: ALT Alanine transaminase, C3 and C4 Complement components, GT Genotype, HCV Hepatitis C virus, RF Rheumatoid factor, n.v. Normal values

chemotherapy 2 years before that had resulted in CR. Finally, two patients who had previously received a diagnosis of $\mathrm{HCC}$ were treated with locoregional ablation therapies with no evidence of recurrence on the basis of contrast-enhanced ultrasound and computed tomography, both before starting therapy and following its completion. At FibroScan evaluation, 12 patients showed advanced liver fibrosis (6 with F3 and 6 with F4 fibrosis and no signs of decompensation), whereas an F1 or F2 score was recorded in the remaining 10 patients.

Four weeks after starting therapy, HCV RNA determination was found to be almost invariably below the lower limit of detection. A rapid and progressive reduction of ALT levels until normalisation was also observed. These strikingly positive results were confirmed throughout the length of treatment. Consequently, all patients reached an EoT response and SVR12 (Fig. 1a) in step with persistent 

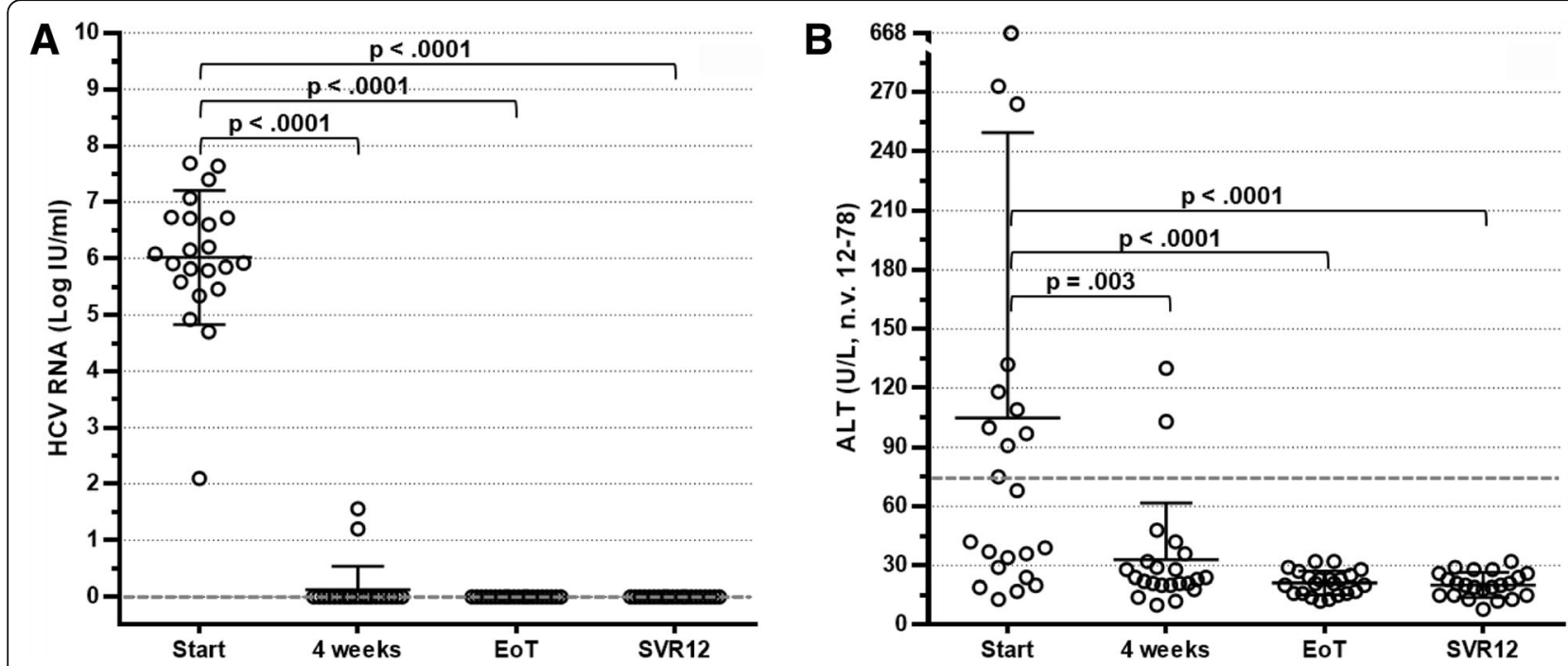

Fig. 1 Distribution of hepatitis C virus (HCV) RNA (a) and alanine transaminase (ALT) (b) levels in 22 HCV-positive patients with cryoglobulinaemia treated with direct-acting antiviral agents at baseline, at week 4, at the end of treatment (EoT) and 12 weeks after treatment. SVR12 Sustained virological response 12 weeks after therapy completion

ALT normalisation (Fig. 1b). Interestingly, seven patients with genotype 1 infection who were given suboptimal SOF/RBV combination therapy also showed SVR12.

An immunological response was observed at EoT in 18 patients $(81.8 \%)$. In 12 patients $(54.5 \%)$, cryoglobulins completely disappeared, whereas in 6 patients $(27.3 \%)$, a cryocrit reduction $\geq 50 \%$ of basal values was observed (Fig. 2a). A less remarkable reduction in cryocrit was calculated in two additional patients, and roughly unchanged levels were observed in the remaining two patients. At the time of SVR12, an overall immunological response was recorded in 17 patients (77.3\%). More precisely, cryoglobulins were undetectable in 13 of them and were reduced to $<50 \%$ of basal value in 4 patients (Fig. 2a). Conversely, cryoglobulins were unchanged in three of the remaining five patients at SVR12, and two patients had relapsed at EoT evaluation.

Additional immunological features also showed a remarkable improvement. As shown in Fig. 2b, test results for RF activity were negative in 12 patients at EoT (54.5\%) and in 11 patients at SVR12 (50\%). In two patients, RF levels were undetectable at EoT but were increased again at SVR12, whereas in one patient the RF test result was negative only at SVR12. Serum C4 levels increased to the normal range in 10 patients at EoT (45.5\%) and in 13 patients at SVR12 (59.1\%) (Fig. 2c).

Compared with basal parameters, cryocrit values were significantly decreased at EoT and SVR12 $(p<0.0001$ and $p=0.001$, respectively) (Table 2 ). The decrease in ALT levels $(p<0.0001$ and $p<0.0001$, respectively) and the normalisation of C4 levels ( $p=0.003$ and $p=0.002$, respectively) also reached statistical significance. Conversely, RF activity was found to be significantly reduced at EoT but not at SVR12 $(p=0.003$ and $p=0.083$, respectively). The results of univariate analysis were statistically significant for all parameters. In addition, considering the effect size value $\left(\eta^{2}\right)$, a remarkable effect of therapy was observed for all biochemical parameters, the effect being particularly prominent for cryocrit and ALT levels $\left(\eta^{2}=0.470\right.$ and $\eta^{2}=0.572$, respectively $)$. These results were confirmed by cross-tabs and $x^{2}$ tests, in that the disappearance of cryoglobulins and ALT normalisation were strongly influenced by therapy (Fig. 3).

In terms of clinical manifestations, we observed regression of Meltzer's triad of symptoms (cryoglobulinaemic glomerulonephritis and peripheral neuropathy), which either were no longer present or were remarkably reduced in 20 patients (90.9\%) and 16 patients $(72.7 \%)$ at EoT and SVR12, respectively. Two patients with peripheral neuropathy had only a partial benefit during antiviral treatment and showed progressive deterioration after EoT. One patient with glomerulonephritis experienced a worsening of renal function at the time of SVR12, but progression of glomerulonephritis to end-stage kidney disease was not observed. Another patient complained of persistent purpuric rashes throughout the treatment and the follow-up period. The patient with SLL treated with DAAs experience a tumour progression that required chemotherapy with fludarabine and cyclophosphamide in addition to RTX. No recurrence or disease progression was recorded in the two patients with previously treated HCC.

According to the above-mentioned response criteria at the time of EoT and SVR12, a CR was observed in 17 patients $(77.3 \%)$ and in 14 patients $(63.7 \%)$, respectively, and a partial virologic plus clinical or immunological response was seen in 4 patients $(18.2 \%)$ and 5 patients 


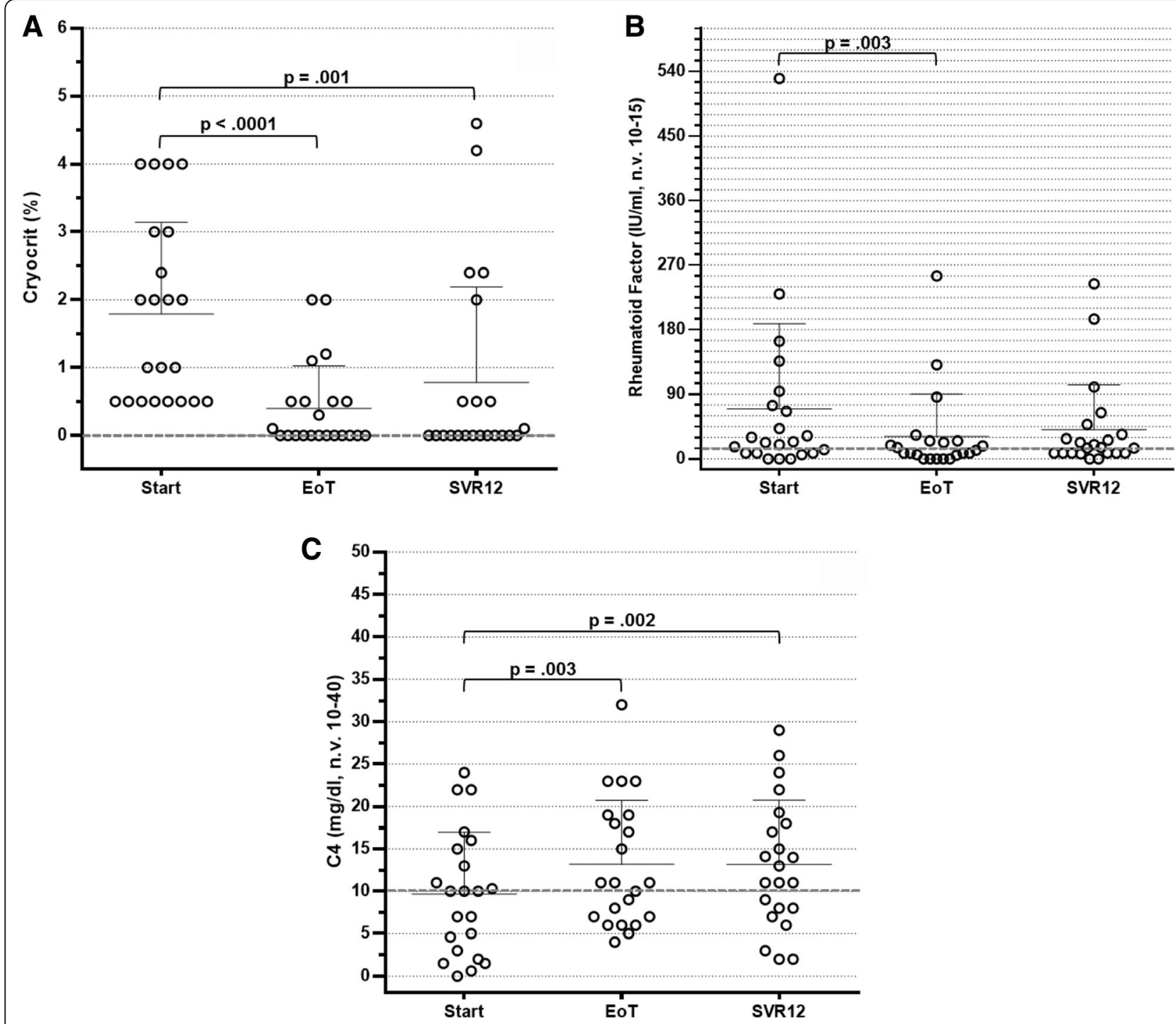

Fig. 2 Distribution of cryocrit levels (a), rheumatoid factor activity (b) and complement component C4 levels (c) in 22 hepatitis C virus-positive patients with cryoglobulinaemia treated with direct-acting antiviral agents at baseline, at week 4, at the end of treatment (EoT) and 12 weeks after treatment. SVR12 Sustained virological response 12 weeks after therapy completion

(22.7\%), respectively. Also at the time of EoT and SVR12, one patient (4.5\%) and three patients (13.6\%), respectively, achieved only SVR. At SVR12, a CR was obtained in 8 of 14 patients receiving SOF/RBV, in all 4 patients receiving SOF/LDV, and in 2 of the 3 patients receiving $3 \mathrm{D}$ combo therapeutic regimens. At the same time point, a PR was observed in 3 of the 14 patients receiving SOF/RBV, in the only patient receiving SOF/ $\mathrm{DCV}$, and in 1 of the 3 patients receiving 3D combo. Three of the fourteen patients who were given SOF/RBV

Table 2 Statistical analysis of the changes in the major biochemical parameters

\begin{tabular}{|c|c|c|c|c|c|c|c|c|}
\hline \multirow[t]{2}{*}{ Parameters } & \multirow{2}{*}{$\begin{array}{l}\text { Baseline } \\
\text { Mean } \pm \text { SD (range) }\end{array}$} & \multicolumn{2}{|l|}{ End of treatment } & \multicolumn{2}{|l|}{ SVR12 } & \multicolumn{3}{|c|}{ Univariate analysis } \\
\hline & & Mean \pm SD (range) & $p$ Value & Mean \pm SD (range) & $p$ Value & $p$ Value & $\eta^{2}$ & Powe \\
\hline Cryocrit, \% & $1.8 \pm 1.3(0.5-4)$ & $0.4 \pm 0.6(0-2)$ & $<0.0001$ & $0.8 \pm 1.4(0-4.6)$ & 0.001 & $<0.0001$ & 0.470 & 0.999 \\
\hline Rheumatoid factor, IU/L & $69.3 \pm 119.1(0-530)$ & $30.9 \pm 58.8(0-255)$ & 0.003 & $40.1 \pm 63.0(0-244)$ & 0.083 & 0.002 & 0.281 & 0.913 \\
\hline $\mathrm{C} 4, \mathrm{mg} / \mathrm{dl}$ & $9.6 \pm 7.3(0-24)$ & $13.6 \pm 8.8(4-36)$ & 0.003 & $14.1 \pm 9.4(2-40)$ & 0.002 & 0.001 & 0.313 & 0.962 \\
\hline $\mathrm{ALT}, \mathrm{IU} / \mathrm{L}$ & $104.8 \pm 144.7(13-668)$ & $20.9 \pm 5.6(12-32)$ & $<0.0001$ & $21.1 \pm 6.6(8-32)$ & $<0.0001$ & $<0.0001$ & 0.572 & 1.000 \\
\hline
\end{tabular}

Abbreviations: ALT Alanine transaminase, C4 Complement component C4, SVR12 Sustained virological response 12 weeks after therapy completion 


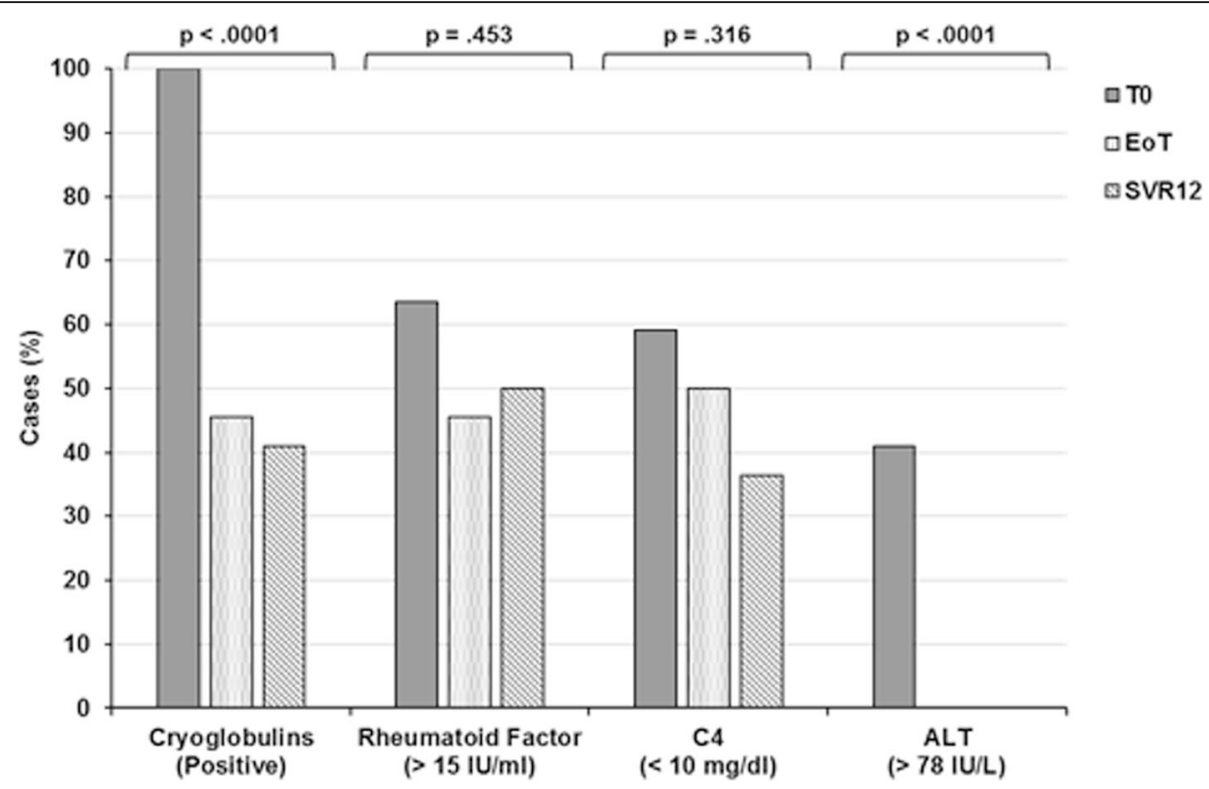

Fig. 3 Cross-tab analysis and $x^{2}$ tests showing modifications of cryocrit, rheumatoid factor (RF), C4 and ALT levels in relation to antiviral therapy. Besides hepatitis C virus RNA, cryocrit and ALT values were significantly influenced by therapy with respect to RF and C4 ( $p<0.0001$ and $p<0.0001$, respectively). ALT Alanine transaminase, C4 Complement component C4, EOT End of treatment, SVR12 Sustained virological response 12 weeks after therapy completion

achieved SVR only. Given that all patients reached an SVR 12 weeks after stopping therapy, 86.4\% of patients in our series obtained a clinical and/or immunological benefit.

Comparing these results with those of our previous study in which we assessed the response to pIFN- $\alpha+$ RBV + RTX combination therapy [19], we found that the all-oral therapeutic approach was significantly better in terms of CR achievement $(p=0.0008$ and $p=0.030$ at EoT and SVR12, respectively) (Table 3).

All patients enrolled in the present study were kept under follow-up, with the aim of assessing the persistence of SVR and the possible changes of clinical and immunological features. At the time of this writing, 20 patients (90.9\%) have achieved SVR24. Among them, cryoglobulins reappeared in three patients (including the patient with SLL), despite the persistence of SVR24 and in the absence of clinical relapse. In one patient, the test for cryoglobulins was negative at EoT, was positive again at SVR12 and then disappeared at SVR24. So far, 6 of the 14 CR patients at SVR12 have been followed for 48 weeks after completion of therapy and have maintained a CR. In two of them, cryoglobulins were absent, although RF activity was still present at levels less than baseline. In two additional patients, the cryocrit was reduced at roughly $50 \%$ of basal value since EoT and remained unchanged throughout follow-up. In the last two patients, both cryocrit and RF remained undetectable. Moreover, one patient with PR and persistence of symptomatic neuropathy despite undetectable cryoglobulins and two SVR-only responsive patients with symptomatic cryoglobulinaemia reached 48 weeks of follow-up.

No severe adverse events related to the administration of DAAs were observed. Two patients receiving SOF/RBV developed progressive anaemia that required reduction of the RBV dosage and erythropoietin administration. Fatigue and mild pain of the dorsolumbar region were

Table 3 Response rates in 22 patients with mixed cryoglobulinaemia following treatment with direct-acting antiviral agents at the end of treatment and sustained virological response 12 weeks after therapy

\begin{tabular}{|c|c|c|c|c|c|c|c|}
\hline \multirow{2}{*}{$\begin{array}{l}\text { Type of } \\
\text { response }\end{array}$} & \multirow{2}{*}{$\begin{array}{l}\text { plFN-a/RBV [19], } \\
n(\%)\end{array}$} & \multicolumn{6}{|c|}{ DAAs therapy, $n$ (\%) } \\
\hline & & EOT & $p$ Value & Power & SVR12 & $p$ Value & Power \\
\hline Complete & $5(33.3)$ & $17(77.3)$ & 0.0008 & 0.895 & $14(63.7)$ & 0.030 & 0.867 \\
\hline Partial & $5(33.3)$ & $4(18.2)$ & 0.216 & 0.801 & $5(22.7)$ & 0.553 & 0.895 \\
\hline SVR only & $5(33.3)$ & $1(4.5)$ & 0.010 & 0.926 & $3(13.6)$ & 0.102 & 0.830 \\
\hline
\end{tabular}

Abbreviations: DAA Direct-acting antiviral agent, EOT End of treatment, pIFN- $a$ Pegylated interferon-a, RBV Ribavirin, SVR12 Sustained virological response 12 weeks after therapy completion

Results achieved with pIFN-a/RBV regimen are given by comparison 
reported by several patients, but in none of them was treatment discontinuation required.

\section{Discussion}

Following the identification of $\mathrm{HCV}$ as the largely prevalent etiologic agent of $\mathrm{MC}$, researchers in several studies have evaluated the therapeutic efficacy of antiviral drugs such as PIFN- $\alpha$, with or without RBV, in this condition [14-16]. These studies consistently reported lower rates of SVR in HCV-positive patients with than in those without MC. In addition, IFN-based regimens were characterised by severe adverse events, often leading to therapy discontinuation. In consideration of the underlying B-cell clonal expansion that characterises MC, B-cell depletion therapy with RTX has also been employed after or in addition to the antiviral treatment [17-19]. MC can indeed be considered as an indolent, benign, lymphoproliferative disease potentially susceptible of evolving into malignant B-NHL [8].

The introduction into clinical practice of the new DAAs for therapy of $\mathrm{HCV}$ infection offers for the first time the possibility of achieving SVR in $>90 \%$, and virtually all, of the chronically infected patients [21], with the coveted perspective of viral eradication and clinical benefits also in patients with extrahepatic manifestations of $\mathrm{HCV}$ infection such as MC.

In this study, we report a single-centre experience with the use of the new DAAs in the therapy of HCV-related MC. The first striking result to be emphasised is the high rate of SVR12. All patients reached this primary endpoint, a dramatic reduction of viraemia starting from the fourth week of therapy. A slightly lower response rate has been demonstrated in patients with MC than in those obtained in patients without cryoglobulinaemia with chronic HCV infection $[24,26]$. The suboptimal efficacy of the SOF/RBV combination employed in those studies has been considered as a possible factor accounting for the reduced SVR rates. At variance from these observations, all seven of our patients with genotype 1 $\mathrm{HCV}$ infection who were treated with SOF/RBV regimen reached SVR12. Gragnani et al. [26] similarly reported $100 \%$ SVR12 and SVR24 by using different SOF-based antiviral combinations, confirming the very high antiviral efficacy of these drugs. More recently, SVR12 was found in $94 \%$ of 64 patients with MC treated with DAAs [27].

As reported above, at SVR12, cryoglobulins disappeared or decreased by at least $50 \%$ in $77.3 \%$ of our patients, whereas RF levels normalised in 50\% and C4 in 63.6\% of the patients. Furthermore, statistical analysis (i.e., univariate analysis, cross-tabs and $X^{2}$ test) showed that cryocrit and ALT levels were the biochemical parameters more significantly influenced by therapy. Because ALT is a biochemical marker of virus-induced hepatocytolysis, its normalisation is obviously expected to follow viral load reduction or disappearance. Similarly, because viral particles are constitutive components of the cryoprecipitating immune complexes, the formation of such complexes is clearly affected when viral particles are no longer present following the administration of DAAs.

On one hand, RF and C4 levels are less influenced by DAAs within the short period of 12 weeks after stopping therapy and may represent independent markers not only of clinical activity but also of persistently activated B-cell clones. On the other hand, on the basis of our experience, variable changes of $\mathrm{RF}$ and $\mathrm{C} 4$ levels may occur when a more prolonged follow-up is taken into consideration, in step with either cryoglobulin disappearance or recurrence.

The administration of DAAs was also shown to result in a remarkable improvement of the clinical manifestations, although symptoms persisted or worsened in six patients (27.3\%) despite their achievement of SVR12 or more. Even so, the overall results ( $63.7 \%$ of CR plus $22.7 \%$ of PR) were significantly better than those obtained with pIFN- $\alpha+$ RBV therapy [19] or with a triple-combination therapy that included first-generation protease inhibitors [20], with the additional advantage of less frequent and less severe side effects in patients receiving DAAs.

Sollima et al. [32] reported a clinical response at week 12 after treatment in two of seven HCV-positive patients with cryoglobulinaemia who were given all-oral antiviral therapy, but one of them had relapsed vasculitis 8 weeks later. It can be inferred that in some cases the pathogenetic process underlying $\mathrm{MC}$ progresses despite viral clearance and that, when a "point of no return" has been overstepped, the immune system keeps synthesising pathogenic virus-independent immune complexes. At the same time, when organ damage appears well established, regression cannot be reasonably expected following antiviral therapy. In our study, only a partial benefit was observed in patients with peripheral neuropathy. Renal function worsened but did not reach end-stage renal disease in one patient with cryoglobulinaemic glomerulonephritis, and vasculitic skin lesions persisted in another patient.

At variance from previous studies in which researchers reported NHL remission following antiviral therapy [33, 34], we observed disease progression in a patient with SLL. Similar observations were reported by Gragnani et al. [26] and more extensively by Arcaini et al. [35], who studied $46 \mathrm{HCV}$-positive patients with indolent NHL treated with IFN-free antiviral regimens and achieved SVR12 in 98\% of the patients and lymphoproliferative disease response of splenic marginal zone lymphomas in 73\% of the patients. On the contrary, NR was reported in patients with chronic lymphocytic leukaemia/SLL. Possible explanations for these findings are that DAAs lack the anti-proliferative and immunomodulatory effects of IFN- $\alpha$ 
and that chronic $\mathrm{HCV}$ infection has a different impact on the oncogenic process in this particular subset of NHLs.

Interestingly, new clinical scenarios are being opened following the treatment with DAAs of patients with chronic HCV infection who have hematologic malignancies [36]. In addition to preventing the development of some NHLs, the use of DAAs in fact may avoid HCV reactivation and hepatic flare after chemotherapy, may induce a better outcome after allogeneic bone marrow transplantation, and may reduce the risk of hepatic failure due to cytotoxic drugs, especially in patients with liver cirrhosis [36].

\section{Conclusions}

Although the worldwide experience with the use of DAAs in HCV-positive patients with cryoglobulinaemia is still limited, our single-centre study shows that variable combinations of DAAs are able to induce SVR12 and SVR24 in virtually all patients. At 12 weeks after EoT, CR and PR were diagnosed in as many as $86.4 \%$ of the patients. These results not only are strikingly better than those obtained with previous antiviral treatments [14-16, 20, 37] but also are closely comparable, in terms of SVR12, to the results reported in remarkably more numerous multicentre cohorts of patients with chronic HCV infection without MC [22, 23].

However, in step with the remarkably high rates of virus eradication induced by the growing cluster of DAAs, new and unforeseen scenarios are emerging. In a minority of patients, clinical and immunological features of cryoglobulinaemic vasculitis persist despite viral clearance. The long-term evolution of these features is totally unknown, and whether an early antiviral approach with DAAs, before the occurrence of severe organ damage, might be able to prevent the occurrence of these initially virus-induced and then virus-cleared vasculitides remains to be elucidated. In addition, at variance from patients with MC achieving SVR and a successful vasculitic response, in whom the regression of B-cell monoclonal expansion in the bone marrow has been described [19], the presence of circulating RF may be associated with the over-representation of mature activated memory $\mathrm{B}$ cells, which persist at least during the early phase following eradication of HCV [38]. To ascertain whether an $\mathrm{HCV}$-independent, B-cell lymphoproliferative disorder may eventually appear in this particular group of patients, more prolonged follow-up after viral clearance is necessary.

\footnotetext{
Abbreviations

3D combo: Ombitasvir/paritaprevir/ritonavir plus dasabuvir; AIFA: Italian Medicines Agency; ALT: Alanine transaminase; B-NHL: B-cell non-Hodgkin lymphoma; CR: Complete response; DAA: Direct-acting antiviral agent; DCV: Daclatasvir; EOT: End of treatment; GLM: General linear model; GT: Genotype; HCC: Hepatocellular carcinoma; HCV: Hepatitis C virus; IFN: Interferon; Ig: Immunoglobulin; LDV: Ledipasvir; MC: Mixed
}

cryoglobulinaemia; NHL: Non-Hodgkin lymphoma; NR: No response; pIFN: Pegylated interferon; PR: Partial response; RBV: Ribavirin; RF: Rheumatoid factor; RTX: Rituximab; SLL: Small lymphocytic lymphoma; SOF: Sofosbuvir; SVR: Sustained virological response; SVR12: Sustained virological response 12 weeks after therapy completion; SVR24: Sustained virological response 24 weeks after therapy completion

\section{Acknowledgements}

Not applicable.

\section{Funding}

This study was supported in part by Associazione Italiana per la Ricerca sul Cancro (AIRC) (investigator grant 14095 [to AV]), the University of Bari "Aldo Moro" (to GL) and Fondo di Sviluppo e Coesione 2007-2013 - APQ Ricerca Regione Puglia "Programma regionale a sostegno della specializzazione intelligente e della sostenibilità sociale ed ambientale - FuturelnResearch" (to SR).

\section{Availability of data and materials}

The datasets used and/or analysed during the present study are available from the corresponding author on reasonable request.

\section{Authors' contributions}

GL, SR, FP and FD substantially contributed to the conception of the study, the analysis and interpretation of the data, and the drafting of the manuscript. AV critically revised the manuscript for important intellectual content. All authors read and approved the final manuscript.

\section{Competing interests}

The authors declare that they have no competing interests.

\section{Consent for publication}

Not applicable.

\section{Ethics approval and consent to participate}

Written informed consent was obtained from all patients. Treatments were administered on-label. Because of the nature of the study, the institutional independent ethical committee of "Azienda Ospedaliero-Universitaria Consorziale Policlinico di Bari" waived the need for ethical approval. The study was conducted according to the principles of the Declaration of Helsinki.

\section{Publisher's Note}

Springer Nature remains neutral with regard to jurisdictional claims in published maps and institutional affiliations.

Received: 14 December 2016 Accepted: 9 March 2017

Published online: 08 April 2017

\section{References}

1. Hajarizadeh B, Grebely J, Dore GJ. Epidemiology and natural history of HCV infection. Nat Rev Gastroenterol Hepatol. 2013;10:553-62.

2. Zeremski M, Dimova RB, Pillardy J, de Jong YP, Jacobson IM, Talal AH. Fibrosis progression in patients with chronic hepatitis $C$ virus infection. J Infect Dis. 2016;214:1164-70.

3. Vescovo T, Refolo G, Vitagliano G, Fimia GM, Piacentini M. Molecular mechanisms of hepatitis $C$ virus-induced hepatocellular carcinoma. Clin Microbiol Infect. 2016:22:853-61.

4. Lanini S, Easterbrook PJ, Zumla A, Ippolito G. Hepatitis C: global epidemiology and strategies for control. Clin Microbiol Infect. 2016;22:833-8.

5. Cacoub P, Comarmond C, Domont F, Savey L, Desbois AC, Saadoun D. Extrahepatic manifestations of chronic hepatitis C virus infection. Ther Adv Infect Dis. 2016:3:3-14

6. Agnello V, Chung RT, Kaplan LM. A role for hepatitis C virus infection in type II cryoglobulinemia. N Engl J Med. 1992;327:1490-5.

7. Dammacco F, Sansonno D. Antibodies to hepatitis $C$ virus in essential mixed cryoglobulinaemia. Clin Exp Immunol. 1992;87:352-6.

8. Dammacco F, Sansonno D, Piccoli C, Racanelli V, D'Amore FP, Lauletta G. The lymphoid system in hepatitis $C$ virus infection: autoimmunity, mixed cryoglobulinemia, and overt B-cell malignancy. Semin Liver Dis. 2000;20:143-57. 
9. Dammacco F, Racanelli V, Russi S, Sansonno D. The expanding spectrum of HCV-related cryoglobulinemic vasculitis: a narrative review. Clin Exp Med. 2016;16:233-42.

10. Lauletta G, Russi S, Conteduca V, Sansonno L. Hepatitis C virus infection and mixed cryoglobulinemia. Clin Dev Immunol. 2012;2012:502156.

11. Lauletta G, Russi S, Conteduca V, Sansonno L, Dammacco F, Sansonno D. Impact of cryoglobulinemic syndrome on the outcome of chronic hepatitis C virus infection. Medicine (Baltimore). 2013;92:245-56.

12. Bonomo L, Casato M, Afeltra A, Caccavo D. Treatment of idiopathic mixed cryoglobulinemia with alpha interferon. Am J Med. 1987;83:726-30.

13. Casato M, Laganà $B$, Antonelli G, Dianzani F, Bonomo L. Long-term results of therapy with interferon-a for type II essential mixed cryoglobulinemia. Blood. 1991;78:3142-7.

14. Dammacco F, Sansonno D. Therapy for hepatitis C virus-related cryoglobulinemic vasculitis. N Engl J Med. 2013;369:1035-45.

15. Cacoub P, Saadoun D, Limal N, Sene D, Lidove O, Piette JC. PEGylated interferon alfa- $2 \mathrm{~b}$ and ribavirin treatment in patients with hepatitis $C$ virusrelated systemic vasculitis. Arthritis Rheum. 2005;52:911-5.

16. Mazzaro C, Monti G, Saccardo F, Zignego AL, Ferri C, De Vita S, et al. Efficacy and safety of peginterferon alfa-2b plus ribavirin for HCV-positive mixed cryoglobulinemia: a multicentre open-label study. Clin Exp Rheumatol. 2011;29:933-41.

17. Sansonno D, De Re V, Lauletta G, Tucci FA, Boiocchi M, Dammacco F. Monoclonal antibody treatment of mixed cryoglobulinemia resistant to interferon a with an anti-CD20. Blood. 2003;101:3818-26.

18. Zaja F, De Vita S, Mazzaro C, Sacco S, Damiani D, De Marchi G, et al. Efficacy and safety of rituximab in type II mixed cryoglobulinemia. Blood. 2003;101:3827-34

19. Dammacco F, Tucci FA, Lauletta G, Gatti P, De Re V, Conteduca V, et al. Pegylated interferon-a, ribavirin, and rituximab combined therapy of hepatitis C virus-related mixed cryoglobulinemia: a long-term study. Blood 2010;116:343-53.

20. Saadoun D, Resche Rigon M, Pol S, Thibault V, Blanc F, Pialoux G, et al. PegIFNa/ribavirin/protease inhibitor combination in severe hepatitis $C$ virusassociated mixed cryoglobulinemia vasculitis. J Hepatol. 2015;62:24-30.

21. Zhang J, Nguyen D, Hu KQ. Chronic hepatitis C virus infection: a review of current direct-acting antiviral treatment strategies. N Am J Med Sci (Boston). 2016:9:47-54.

22. European Association for the Study of the Liver. EASL recommendations on treatment of hepatitis C 2016. J Hepatol. 2017:66:153-94.

23. AASLD/IDSA HCV Guidance Panel. Hepatitis C guidance: AASLD-IDSA recommendations for testing, managing, and treating adults infected with hepatitis C virus. Hepatology. 2015;62:932-54.

24. Saadoun D, Thibault V, Si Ahmed SN, Alric L, Mallet M, Guillaud C, et al. Sofosbuvir plus ribavirin for hepatitis C virus-associated cryoglobulinaemia vasculitis: VASCUVALDIC study. Ann Rheum Dis. 2016;75:1777-82.

25. Sise ME, Bloom AK, Wisocky J, Lin MV, Gustafson JL, Lundquist AL, et al. Treatment of hepatitis $C$ virus-associated mixed cryoglobulinemia with direct-acting antiviral agents. Hepatology. 2016;63:408-17.

26. Gragnani L, Visentini M, Fognani E, Urraro T, De Santis A, Petraccia L, et al. Prospective study of guideline-tailored therapy with direct-acting antivirals for hepatitis C virus-associated mixed cryoglobulinemia. Hepatology. 2016:64:1473-82

27. Bonacci M, Lens S, Londoño MC, Mariño Z, Cid MC, Ramos-Casals M, et al. Virologic, clinical, and immune response outcomes of patients with hepatitis $C$ virus-associated cryoglobulinemia treated with direct-acting antivirals. Clin Gastroenterol Hepatol. doi:10.1016/j.cgh.2016.09.158.

28. Quartuccio L, Isola M, Corazza L, Ramos-Casals M, Retamozo S, Ragab GM, et al. Validation of the classification criteria for cryoglobulinaemic vasculitis. Rheumatology (Oxford). 2014;53:2209-13.

29. Brouet JC, Clauvel JP, Danon F, Klein M, Seligmann M. Biologic and clinical significance of cryoglobulins: a report of 86 cases. Am J Med. 1974;57:775-88

30. Musset L, Diemert MC, Taibi F, Du Thi Huong L, Cacoub P, Leger JM, et al. Characterization of cryoglobulins by immunoblotting. Clin Chem. 1992:38:798-802.

31. Trejo O, Ramos-Casals M, García-Carrasco M, Yagüe J, Jiménez S, de la Red $G$, et al. Cryoglobulinemia: study of etiologic factors and clinical and immunologic features in 443 patients from a single center. Medicine (Baltimore). 2001;80:252-62.
32. Sollima S, Milazzo L, Peri AM, Torre A, Antinori S, Galli M. Persistent mixed cryoglobulinaemia vasculitis despite hepatitis $C$ virus eradication after interferon-free antiviral therapy. Rheumatology (Oxford). 2016;55:2084-5.

33. Hartridge-Lambert SK, Stein EM, Markowitz AJ, Portlock CS. Hepatitis C and non-Hodgkin lymphoma: the clinical perspective. Hepatology. 2012;55:634-41.

34. Arcaini L, Vallisa D, Rattotti S, Ferretti W, Ferreri AJM, Bernuzzi P, et al. Antiviral treatment in patients with indolent B-cell lymphomas associated with HCV infection: a study of the Fondazione Italiana Linfomi. Ann Oncol. 2014:25:1404-10.

35. Arcaini L, Besson C, Frigeni M, Fontaine $H$, Goldaniga M, Casato M, et al. Interferon-free antiviral treatment in B-cell lymphoproliferative disorders associated with hepatitis C virus infection. Blood. 2016;128:2527-32.

36. Torres HA, McDonald GB. How I treat hepatitis $C$ virus infection in patients with hematologic malignancies. Blood. 2016;128:1449-57.

37. Saadoun D, Resche-Rigon M, Thibault V, Piette JC, Cacoub P. Antiviral therapy for hepatitis $C$ virus-associated mixed cryoglobulinemia vasculitis: a long-term follow-up study. Arthritis Rheum. 2006:54:3696-706.

38. Reyes-Avilés E, Kostadinova L, Rusterholtz A, Cruz-Lebrón A, Falck-Ytter Y, Anthony DD. Presence of rheumatoid factor during chronic HCV infection is associated with expansion of mature activated memory B-cells that are hypo-responsive to B-cell receptor stimulation and persist during the early stage of IFN free therapy. PLoS One. 2015:10:e0144629.

\section{Submit your next manuscript to BioMed Central and we will help you at every step:}

- We accept pre-submission inquiries

- Our selector tool helps you to find the most relevant journal

- We provide round the clock customer support

- Convenient online submission

- Thorough peer review

- Inclusion in PubMed and all major indexing services

- Maximum visibility for your research

Submit your manuscript at www.biomedcentral.com/submit
) Biomed Central 